\section{Polyarthritis associated with hydatid disease of the liver}

\author{
Rawdha Tekaya,' Leila Souabni," \\ Chiraz Ben Hadj Yahia,' Imen Sfar, \\ Yosr Gorgi, ${ }^{2}$ Rafik Zouari' \\ 'Department of Rheumatology, Charles \\ Nicolle Hospital, Tunis, Tunisia; ${ }^{2}$ Division \\ of Immunology, Charles Nicolle Hospital, \\ Tunis, Tunisia
}

\section{Abstract}

Clinical presentation of hydatid disease depends on the immunological background of the patient. Articular site of the parasite can give rise either to a true echinococcal arthritis or to a synovial affection that does not depend on living larva. A 77-year old man who had had hydatid disease of the liver for two years, presented with progressive onset arthritis. Laboratory studies showed inflammatory changes but no evidence of immunological disorders was seen. Abdominal imaging revealed multiple hydatid cysts of the liver. Ankle synovial fluid evaluation was positive for antibodies for hydatid antigen and negative for echinococcal larva. Excision of the hydatid cyst was accompanied by full remission of the arthritis with no recurrence. A reactive immune mechanism triggered by a parasite located at a distant side appears to be responsible for this type of arthritis. These data support the potential of echinococcosis granulosus in inducing a true aseptic arthritis as a response to intense immunological disorders.

\section{Introduction}

Arthritis associated with parasitic infestation has been frequently reported in the past. ${ }^{1}$ Reported cases include arthritis associated with infestation by protozoa, platyhelminthes and nemathelminthes. These arthritic symptoms have merited the term "parasitic rheumatism".

The literature of arthritis associated with hydatid cyst (echinococcosis) remains confined to isolated clinical cases, making it difficult to draw conclusions regarding pathophysiology. ${ }^{2,3}$ Herein, we describe an unusual case of a patient with a past history of hydatid disease of the liver who developed parasitic rheumatism which improved after the removal of the echinococcal cyst.

\section{Case Report}

A 77-year old male farmer was admitted for a 4-month history of inflammatory arthralgia and morning stiffness. Comorbidities included diabetes and hydatid disease of the liver for the previous six months for which surgical treatment is indicated but had been declined by the patient. Physical examination revealed stress pain in the right wrist and synovitis of the knees. The left ankle was swollen, hot and painful. Examination revealed a limitation of movement. The liver was enlarged but not tender. His laboratory findings were as follows: erythrocyte sedimentation rate $63 \mathrm{~mm} / \mathrm{h}, \mathrm{C}$ reactive protein $34 \mathrm{mg} / \mathrm{dL}(\mathrm{N}=0-8)$, aspartate aminotransferase $26 \mathrm{U} / \mathrm{L}(\mathrm{N}=0-40)$, alanine aminotransferase $19 \mathrm{U} / \mathrm{L}(\mathrm{N}=0-40)$, serum urea $7 \mathrm{mg} / \mathrm{dL}$ (5-20). There was no peripheral blood eosinophilia or leukocytosis. Rheumatoid factor, antinuclear antibody and antibodies to extractable nuclear antigens were negative. HLA typing was A2 A34 B8 B51 DR2 DR3 DQ1 DQ2. There was no evidence of chondrocalcinosis on plain radiographs. Abdominal ultrasound showed well-circumscribed cystic masses in the liver. Serological examinations were performed with the following results: positive values on hydatid disease (using ELISA and confirmation with hemagglutination reaction), negative values on toxocariasis and cysticercosis (ELISA in both). Ankle synovial fluid evaluation showed an absolute cell count of $25,000 / \mathrm{mm}^{3}$ with $73 \%$ polymorphonuclear leukocytes, $27 \%$ lymphocytes. Crystal analysis fluid and micro-organism results were negative, but we identified hydatid antigen in the synovial fluid. Synovial biopsy excluded local synovial infection and crystal synovitis. Treatment with different nonsteroidal anti-inflammatory drugs proved ineffective. Surgical excision of the liver cysts along with chemotherapy (albendazole) was then performed. Two months later, the patient's left ankle improved and progressively recovered its normal function. A regular control by echinococcosis serology was negative. More than one year later, the patient is still free of articular symptoms.

\section{Discussion}

We present a case of acute oligoarticular inflammatory arthritis (wrist, knees and ankle) in a patient with known hepatic hydatid disease. The assumption is then made that this is a parasitic arthritis. Moreover, there is a temporal relationship with subsequent treatment of the liver disease (surgery and pharmacological therapy). Another possibility might be local infection. Although much rarer than
Correspondence: Rawdha Tekaya,

Department of Rheumatology, Charles Nicolle Hospital, Tunis, Tunisia

E-mail: tekayarawdha@yahoo.fr

Key words: polyarthritis, reactive arthritis, hydatid cyst, echinococcus, parasitic diseases.

Conflict of interest: the authors reported no potential conflicts of interests.

Received for publication: 18 April 2009.

Revision received: 24 June 2009.

Accepted for publication: 29 June 2009

This work is licensed under a Creative Commons Attribution 3.0 License (by-nc 3.0)

(C) Copyright R. Tekaya et al., 2009

Licensee PAGEPress, Italy

Rheumatology Reports 2009; 1:e2

doi:10.4081/rr.2009.e2

crystal synovitis, such a presentation of large/medium joints has been described previously ${ }^{4}$ as these authors demonstrate synovial infiltration of echinococcus. In the current case report, we identify hydatid antigen in the synovial fluid but synovial biopsy excluded local synovial infection. The described patient's symptoms are improved following excision of the hepatic cyst and pharmacological treatment (albendazole) for the liver supporting the hypothesis of a reactive arthritis. In endemic areas, clinical presentation of hydatid disease depends on immunological background of the patient. Articular site of the parasite can give rise either to a true echinococcal arthritis or to a synovial affection that does not depend on living larva. Different immunological studies performed in patients with hydatidosis and in experimental animals infected with E. granulosus led to observations that would explain the perpetuation of the infection by the continuous liberation of antigen which lead to the formation of low levels of small molecular size immune complexes. ${ }^{5}$ Even though the parasitic antigen has rarely been demonstrated in the involved organ, ${ }^{6}$ a relationship between clinical symptoms and the remission of biological disorders after surgical removal of the hydatid cyst is indirect but strong evidence of its pathogenic mechanism. To explain the uncommon occurrence of this variety of reactive arthritis due to parasitic infestation, despite the high prevalence of parasitic infestation in the world, a hypothesis of genetic predisposition seems valuable. Among 34 well documented reported cases of parasitic rheumatism in the literature, HLA B27 antigen was researched in 13; of these, HLA B27 was absent in 9 . In 7 of these 9, the clinical picture was symmetrical polyarthritis. When HLA B27 was present ( 4 cases), the clinical picture is 
asymmetrical pauci-arthritis, mimicking arthritis of Reiter's disease. This patient was HLA B27 negative.

Even in endemic areas, such presentations are relatively rare and parasitic infestation may underlie the clinical presentation of some rheumatic conditions. In these cases, parasitic infections have to be excluded because suppression of the host's immune system could be associated with lethal disseminated infection. We suggest that echinococcus infection should be considered, especially in countries where hydatid disease is endemic.

\section{References}

1. Doury P. Parasitic rheumatism. Arthritis Rheum 1981;24:638-9.

2. Buskila D, Sukenik S, Klein M, Horowitz J. Polyarthritis associated with hydatid disease (echinococcosis) of the liver. Clin Rheumatol 1992;2:286-7.

3. Weill BJ, Grelier JL, Menkès CJ, Delaitre B. Polyarthritis associated with hydatid cyst of the liver. Rev Rhum Mal Osteoartic 1988;55:629.

4. Panagiotis G. Vallianatos, Anastasia C, et al. Echinococcal synovitis of the knee joint. Arthroscopy 2002;18:E48.

5. D'Amelio R, Pontesilli 0, Palmisano L, et al. Detection and partial characterization of circulating immune complexes in hydatid disease. J Clin Microbiol 1983;18:1021-6.

6. Sánchez Ibarrola A, Sobrini B, Guisantes J, et al. Membranous glomerulonephritis secondary to hydatid disease. Am J Med 1981;70:311-5.

7. Bakkalo€u A, Söylemezo€u 0, Tinaztepe K, Saatci U. A possible relationship between polyarteritis nodosa and hydatid disease. Eur J Pediatr 1994;153:469. 